\title{
Multicomponent Electron-Hole Superfluidity and the BCS-BEC Crossover in Double Bilayer Graphene
}

\author{
S. Conti, ${ }^{1,2}$ A. Perali, ${ }^{1, *}$ F. M. Peeters, ${ }^{2}$ and D. Neilson ${ }^{1,2}$ \\ ${ }^{1}$ Dipartimenti di Fisica e di Farmacia, Università di Camerino, 62032 Camerino (MC), Italy \\ ${ }^{2}$ Department of Physics, University of Antwerp, Groenenborgerlaan 171, B-2020 Antwerpen, Belgium
}

(Received 22 June 2017; published 22 December 2017)

\begin{abstract}
Superfluidity in coupled electron-hole sheets of bilayer graphene is predicted here to be multicomponent because of the conduction and valence bands. We investigate the superfluid crossover properties as functions of the tunable carrier densities and the tunable energy band gap $E_{g}$. For small band gaps there is a significant boost in the two superfluid gaps, but the interaction-driven excitations from the valence to the conduction band can weaken the superfluidity, even blocking the system from entering the Bose-Einstein condensate (BEC) regime at low densities. At a given larger density, a band gap $E_{g} \sim 80-120 \mathrm{meV}$ can carry the system into the strong-pairing multiband BCS-BEC crossover regime, the optimal range for realization of high- $T_{c}$ superfluidity.
\end{abstract}

DOI: 10.1103/PhysRevLett.119.257002

The recent fabrication of two very close, but electrically isolated, conducting bilayer graphene sheets, one containing electrons and the other holes [1-4], raises exciting possibilities of observing high-temperature superfluidity [5], since the electrons form pairs with the holes through very strong Coulomb attraction [6,7].

In bilayer graphene, the Fermi energy can be tuned continuously relative to the average strength of the Coulomb interactions between carriers [5]. Metal gates can be used to change the carrier densities [8] so as to tune each sheet from the high-density regime of weak interactions to the low-density regime, where the average Coulomb interactions between carriers are much larger than their kinetic energies. However, the touching of the conduction and valence bands at the semimetallic point means that at low densities, carriers from the two bands can strongly affect each other, and this weakens the superfluid pairing. A tunable energy band gap inserted between the conduction and valence bands by application of electric fields perpendicular to the sheets [9] can be used to decouple the conduction and valence bands. By contrast, in multicomponent high- $T_{c}$ iron-based superconductors, the carrier densities are difficult to tune and their energy band structure is fixed $[10,11]$.

We investigate the effect of the multibands at zero temperature on the superfluid BCS-BEC crossover and Bose-Einstein condensate (BEC) regimes as functions of the tunable carrier densities and energy band gap $E_{g}$, in order to identify optimal combinations of the experimental parameters for superfluidity. We find that the crossover properties are sensitive to both the carrier densities and the energy band gaps.

Our standard multiband Hamiltonian is specified in Eq. (S1) of the Supplemental Material [12], which is based on Refs. [13,14]. We include intraband pairing and Josephson-like pair transfer between the conduction and valence bands, which are labelled by $\gamma= \pm$. Our neglect of crosspairing is justified later in the Letter. The coupled mean field gap equations at zero temperature for equal electron and hole densities are [13],

$$
\Delta_{k}^{\gamma}=-\sum_{k^{\prime}, \gamma^{\prime}} F_{k k^{\prime}}^{\gamma \gamma^{\prime}} V_{k k^{\prime}} \frac{\Delta_{k^{\prime}}^{\gamma^{\prime}}}{2 E_{k^{\prime}}^{\gamma^{\prime}}},
$$

where $E_{k}^{\gamma}=\sqrt{\left(\xi_{k}^{\gamma}\right)^{2}+\left(\Delta_{k}^{\gamma}\right)^{2}}$ and $\xi_{k}^{\gamma}=\left(\xi_{k}^{(e) \gamma}+\xi_{k}^{(h) \gamma}\right) / 2$. The form factor for the overlap of the single-particle state $|k\rangle$ in band $\gamma$ with $\left|k^{\prime}\right\rangle$ in band $\gamma^{\prime}$ is given by [15]

$F_{\mathbf{k k}^{\prime}}^{\gamma \gamma^{\prime}}=\frac{1}{2}\left[1+\gamma \gamma^{\prime}\left(\cos \alpha_{k} \cos \alpha_{k^{\prime}}+\sin \alpha_{k} \sin \alpha_{k^{\prime}} \cos 2 \phi\right)\right]$,

where $\phi=\cos ^{-1}\left(\widehat{\mathbf{k k}^{\prime}}\right)$ and $\alpha_{k}=\tan ^{-1}\left[\hbar^{2} k^{2} /\left(m^{*} E_{g}\right)\right]$. We note the important dependence of $F_{\mathbf{k k}^{\prime}}^{\gamma \gamma^{\prime}}$ on $E_{g}$.

To determine the chemical potential $\mu$, we take for the density control parameter for each bilayer sheet [16,17],

$$
n_{0}^{+}=g_{s} g_{v} \sum_{k}\left[\left(v_{k}^{+}\right)^{2}-\left(u_{k}^{-}\right)^{2}\right] .
$$

$g_{s}=g_{v}=2$ are the spin and valley degeneracy for bilayer graphene, and the band-dependent Bogoliubov amplitudes are $\left(v_{k}^{\gamma}\right)^{2}=\left(1-\xi_{k}^{\gamma} / E_{k}^{\gamma}\right) / 2$ and $\left(u_{k}^{\gamma}\right)^{2}=\left(1+\xi_{k}^{\gamma} / E_{k}^{\gamma}\right) / 2 \cdot n_{0}^{+}$ is defined as the total number of carriers in the conduction band, $n^{+}=g_{s} g_{v} \sum_{k}\left(v_{k}^{+}\right)^{2}$, less the number of carriers in the conduction band that have been excited from the valence band. The number of such excited carriers in the conduction band equals the number of unoccupied states left behind in the valence band, $g_{s} g_{v} \sum_{k}\left(u_{k}^{-}\right)^{2} . \mu$ is obtained by solving Eqs. (1)-(3). 
The reason for this choice of control parameter [Eq. (3)] is due to the influence of the valence band on the conduction band. The presence of the valence band means that the overall number of carriers in the conduction band, $n^{+}$, is no longer controlled purely by doping or using the metal gates, as is the case for the single band system, since now there are additional carriers in the conduction band excited from the valence band due to interactions. This increase in the number of carriers in the conduction band will push up the Fermi energy. We use $n^{+}$to define an effective Fermi momentum $k_{F}^{*}=\sqrt{4 \pi n^{+} / g_{s} g_{v}}$, and effective Fermi energy in the conduction band $E_{F}^{*}=$ $\left(\hbar k_{F}^{*}\right)^{2} / 2 m^{*}$.

For large $n_{0}^{+}$, the average kinetic energy of the carriers in the conduction band $\langle K\rangle$ is large relative to the average strength of the Coulomb interactions $\langle V\rangle$, and, since $\sum_{k}\left(v_{k}^{+}\right)^{2} \gg \sum_{k}\left(u_{k}^{-}\right)^{2}$, there are only a negligible number of carriers excited out of the valence band. However, small $n_{0}^{+}$does not necessarily imply that $\langle K\rangle \ll\langle V\rangle$, since for sufficiently small $E_{g}$, both $\sum_{k}\left(v_{k}^{+}\right)^{2}$ and $\sum_{k}\left(u_{k}^{-}\right)^{2}$ can be large but nearly equal. We will see that both $n_{0}^{+}$and $E_{g}$ play important roles in determining the relative strength of the Coulomb interactions.

We take the interaction term in Eq. (1) as unscreened,

$$
V_{k k^{\prime}}=-\frac{2 \pi e^{2}}{\epsilon} \frac{e^{-d\left|\mathbf{k}-\mathbf{k}^{\prime}\right|}}{\left|\mathbf{k}-\mathbf{k}^{\prime}\right|} .
$$

Few layers of a hexagonal boron nitride (hBN) insulating barrier with thickness $d \geq 1 \mathrm{~nm}$ act as a good barrier to electrically isolate the two graphene bilayer sheets [18]. We take a barrier thickness $d=1 \mathrm{~nm}$. The barrier height is $\sim 1.5 \mathrm{eV}$. For bilayer graphene encapsulated in few-layer hBN, the dielectric constant $\epsilon \sim 2$ [19].

By comparing the superfluid properties calculated using diffusion quantum Monte Carlo [20] with the results for the same system calculated within mean field without screening, Ref. [21] demonstrated that neglecting screening is an excellent approximation in the BEC regime where the strong interactions tightly bind the pairs, making the pairs compact on the scale of the intercarrier separations $r_{0}$. For example, at density $n_{0}^{+}=1 \times 10^{11} \mathrm{~cm}^{-2}, r_{0}=18 \mathrm{~nm}$ is much larger than the effective Bohr radius in graphene. In the BEC regime, the gaps $\Delta^{\gamma}$ in the single-particle excitation spectrum are much larger than the Fermi energies, and this suppresses the long-wavelength excitations needed for screening [22]. Reference [21] also showed that the unscreened approximation remains surprisingly accurate even in the BCS-BEC crossover regime at intermediate densities [5], predicting superfluid gaps correctly to within $20 \%$ [21]. However, at larger densities, $n_{0}^{+} \gtrsim 5 \times 10^{11} \mathrm{~cm}^{-2}$, in what would have been the BCS regime, the unscreened approximation completely breaks down, since at these densities there is very strong screening which kills the superfluidity $[5,22]$. Thus, we restrict our results to densities $n_{0}^{+} \leq 5 \times 10^{11} \mathrm{~cm}^{-2}$.
We omit the intralayer interactions. This is justified by comparing superfluid gaps calculated including correlations between like species [23], with the gaps calculated neglecting these correlations [24]. The intralayer correlations are found to have a $\lesssim 10 \%-20 \%$ effect on the gaps.

In general, the regimes of the crossover phenomena in a one-band system are conveniently characterized by the superfluid condensate fraction $c$ [25]. $c$ is defined as the fraction of carriers bound in pairs relative to the total number of carriers. The usual classification is: for $c>0.8$ the condensate is in the BEC regime, for $c<0.2$ it is in the $\mathrm{BCS}$ regime, and otherwise it is in the crossover regime.

However, we have here two partial condensate fractions, $c^{ \pm}$, for the conduction and valence bands. For the conduction band the usual one-band expression is readily generalized to the number of pairs divided by the total number of carriers in the conduction band,

$$
c^{+}=\frac{\sum_{k}\left(u_{k}^{+}\right)^{2}\left(v_{k}^{+}\right)^{2}}{\sum_{k}\left(v_{k}^{+}\right)^{2}},
$$

but for the valence band the corresponding definition of $c^{-}$ is the ratio of the number of pairs in the valence band to the number of antiparticles in the valence band,

$$
c^{-}=\frac{\sum_{k}\left(u_{k}^{-}\right)^{2}\left(v_{k}^{-}\right)^{2}}{\sum_{k}\left(u_{k}^{-}\right)^{2}} .
$$

(We use the term antiparticle to refer to an empty singleparticle state in the valence band, since we reserve the term hole to refer to the hole-doped bilayer sheet.) At zero temperature, the valence band antiparticles are generated exclusively as a result of the effect of interactions that excite carriers out of the valence band up into the conduction band. The pairs in the valence band are formed from pairing of antiparticles of the two sheets.

Figure 1 compares the contributions to pair formation from the conduction and valence bands as a function of $n_{0}^{+}$. The ratios $n_{\text {pair }}^{ \pm} /\left(n_{\text {pair }}^{+}+n_{\text {pair }}^{-}\right)$are shown for different energy band gaps, where $n_{\text {pair }}^{\gamma}=\sum_{k}\left(u_{k}^{\gamma}\right)^{2}\left(v_{k}^{\gamma}\right)^{2}$. As expected, for large values of $E_{g}$, pair formation is confined

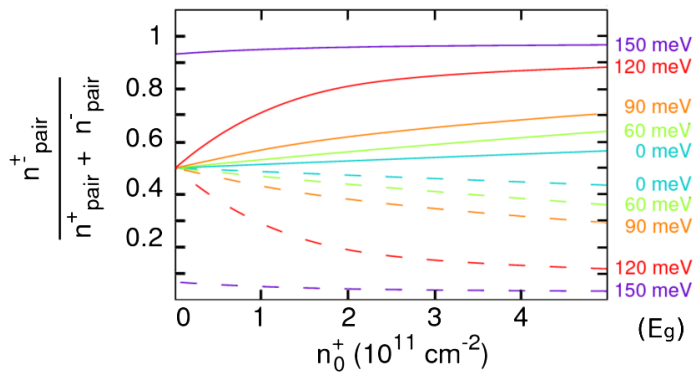

FIG. 1. Relative number of condensate pairs in conduction band (solid lines) and valence band (dashed lines) as functions of $n_{0}^{+}$for different values of energy band gap $E_{g}$. 


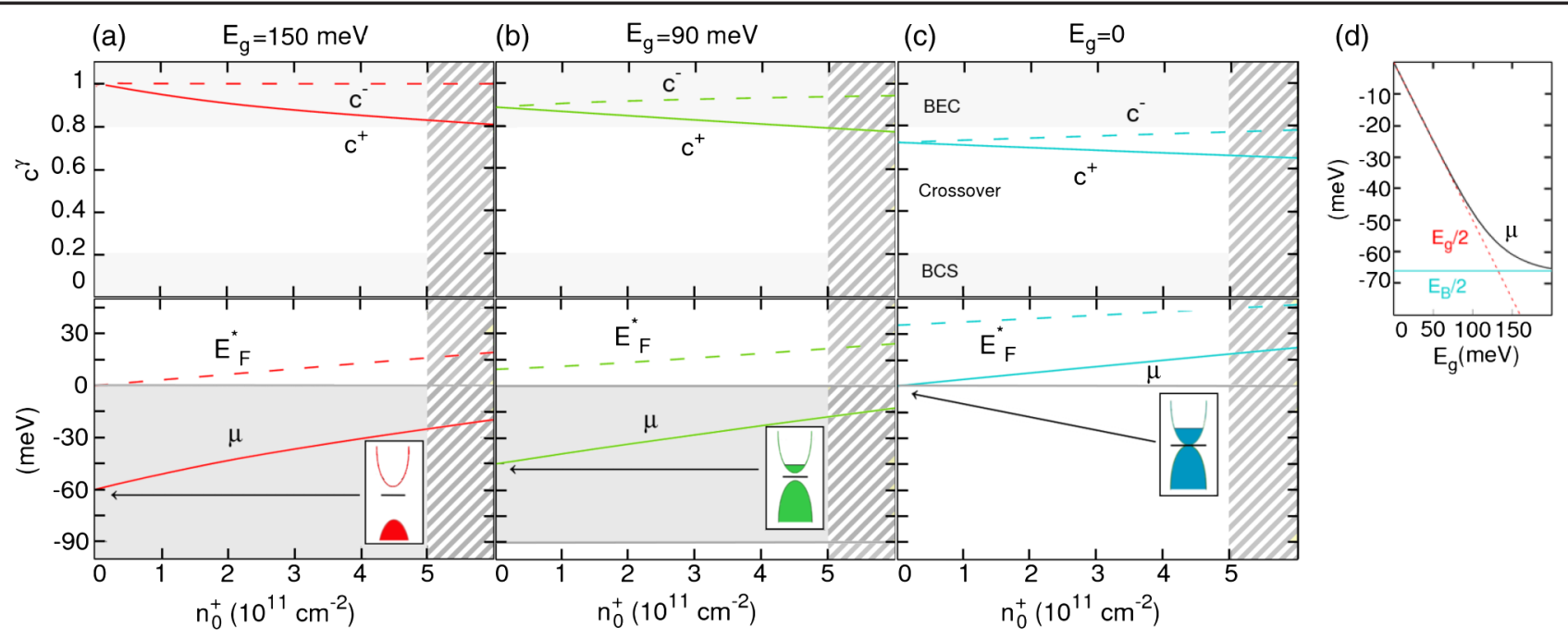

FIG. 2. (a)-(c) Condensate fractions $c^{ \pm}$, chemical potential $\mu$, and effective Fermi energy $E_{F}^{*}$ as functions of $n_{0}^{+}$. Upper panels show condensate fraction in the conduction band (solid lines) and valence band (dashed lines). Lower panels show chemical potential (solid lines) and effective Fermi energy (dashed lines). Gray shaded area is the band gap. Density region $n_{0}^{+}>5.0 \times 10^{11} \mathrm{~cm}^{-2}$ is excluded from our results since inclusion of screening will suppress the superfluidity there. (d) Limiting strong-coupling BEC low-density behavior of chemical potential as a function of $E_{g}$.

to the conduction band and is independent of $n_{0}^{+}$. However, for smaller $E_{g}$, the ratios depend on $n_{0}^{+}$. We recall that large $n_{0}^{+}$signifies a small valence band contribution because it contains few antiparticles, while at small $n_{0}^{+}$both bands contribute equally to the pair formation, whether the interactions are strong or weak.

Figure 2 shows the condensate fractions and the chemical potential as functions of $n_{0}^{+}$. For a large band gap, $E_{g}=$ $150 \mathrm{meV}$ [Fig. 2(a)], the behavior of $c^{ \pm}$and $\mu$ is indeed close to the results for a one-band system [26]. Here, for $n_{0}^{+} \sim 5 \times 10^{11} \mathrm{~cm}^{-2}$, the conduction band condensate is already close to the crossover regime boundary, with $c^{+} \sim 0.8$. We recall for $n_{0}^{+}>5 \times 10^{11} \mathrm{~cm}^{-2}$ screening is expected to suppress superfluidity. As $n_{0}^{+}$decreases, $c^{+}$ becomes $>0.8$, and the conduction band condensate enters the $\mathrm{BEC}$ regime. The chemical potential is everywhere less than $E_{F}^{*}$, and it is negative because we are in the BEC regime. As $n_{0}^{+}$goes to zero, the conduction band condensate enters the deep BEC limit and $\mu \rightarrow-\varepsilon_{B} / 2$, half the binding energy of an independent electron-hole pair [see Fig. 2(d)]. We see that $c^{-} \sim 1$, the valence band condensate remains in the BEC regime over the full range of $n_{0}^{+}$shown, because there are so few antiparticles in the valence band when $E_{g}$ is large.

For a smaller gap, $E_{g}=90 \mathrm{meV}$ [Fig. 2(b)], the conduction band condensate is slower to enter the BEC regime with decreasing $n_{0}^{+}$. This is because excitations from the valence band now significantly increase the total population of carriers in the conduction band. The chemical potential $\mu$ therefore goes negative only at very low $n_{0}^{+}$. It is interesting that in the zero $n_{0}^{+}$limit, $\mu$ now approaches the midpoint of the band gap, $\mu \rightarrow-E_{g} / 2$ instead of $-\varepsilon_{B} / 2$, behavior analogous to the intrinsic limit in a conventional semiconductor.

Figure 2(c) is for $E_{g}=0$. In this case there are many carriers in the conduction band excited from the valence band. This makes the effective Fermi energy $E_{F}^{*}$ as a function of $n_{0}^{+}$significantly larger than in Figs. 2(a) and 2(b), and at the same time a large number of antiparticles are created in the valence band. For this reason, both the conduction and valence band condensates are always in the crossover regime for all $n_{0}^{+}$and the chemical potential $\mu$ remains positive. An interesting point is that for a system with $E_{g}=0$, a negative value of $\mu$ would signify only an inversion of the carrier populations in the bands, so that even for negative values of $\mu$, the system would remain in the crossover regime.

Figure 3 shows the momentum-dependent superfluid energy gaps $\Delta_{k}^{ \pm}$. For large $E_{g}$, Fig. 3(a), the gap equations [Eq. (1)] are nearly decoupled because the $E_{g}$ term in the form factor [Eq. (2)] suppresses $F_{\mathbf{k k}^{\prime}}^{\gamma \gamma^{\prime}}$ for $\gamma \neq \gamma^{\prime}$. Then $\Delta_{k}^{+} \gg \Delta_{k}^{-}$, because of the large energy denominator for $\Delta_{k}^{-}$ in Eq. (1). Consistent with the conclusion in Fig. 2(a), the very broad peaks in $\Delta_{k}^{-}$for all $n_{0}^{+}$indicate that the valence band condensate for large $E_{g}$ always remains in the BEC regime. The reason is that the number of paired antiparticles in the valence band, $\sum_{k}\left(u_{k}^{-}\right)^{2}$, remains small for all $n_{0}^{+}$. For the same reason, the conduction band contains very few carriers excited from the valence band, so the evolution of the conduction band condensate with $n_{0}^{+}$is very similar to the one-band system: i.e., (i) for small $n_{0}^{+}, \Delta_{k}^{+} \gg E_{F}$, its peak is at $k=0$ and it is very broad, which are characteristics of the BEC regime, and (ii) for large $n_{0}^{+}$, the peak in $\Delta_{k}^{+}$becomes of order $E_{F}^{*}$, and it narrows and detaches from 


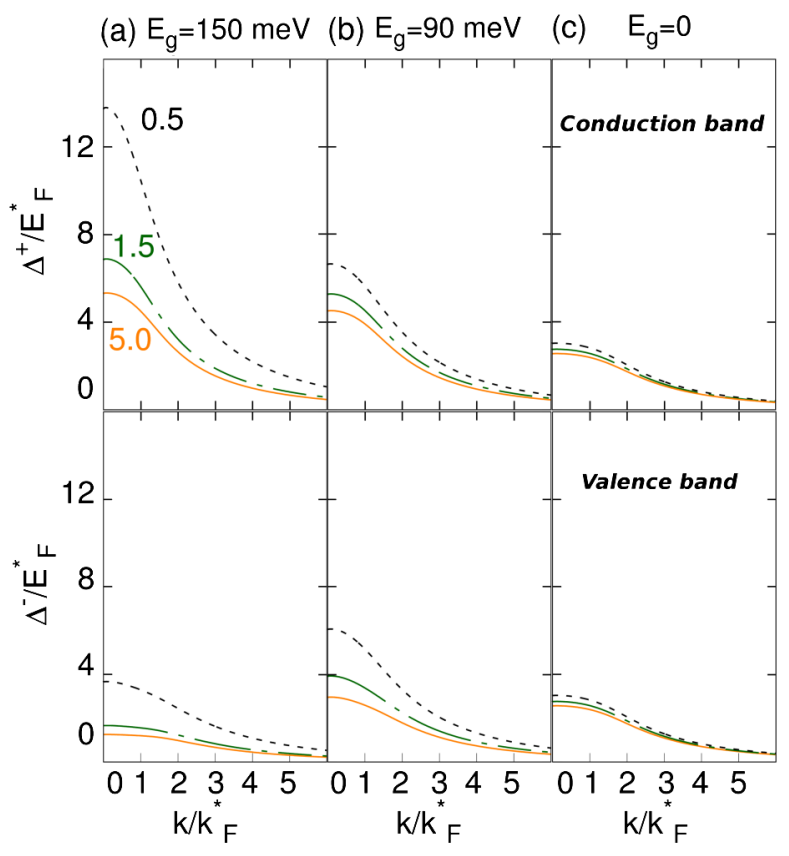

FIG. 3. The superfluid gap energy $\Delta_{k}^{\gamma= \pm}$ in conduction and valence bands. Dotted lines, $n_{0}^{+}=0.5 \times 10^{11} \mathrm{~cm}^{-2}$; dashed lines, $n_{0}^{+}=1.5 \times 10^{11} \mathrm{~cm}^{-2}$; solid lines, $n_{0}^{+}=5 \times 10^{11} \mathrm{~cm}^{-2}$.

$k=0$, though never reaching $k=k_{F}^{*}$, which are characteristics of the crossover regime.

For smaller $E_{g}$, Figs. 3(b) and 3(c), the $\Delta_{k}^{ \pm}$are comparable and are not very sensitive to $n_{0}^{+}$. This is because the $F_{\mathbf{k k}^{\prime}}^{\gamma \gamma^{\prime}}$ for $\gamma \neq \gamma^{\prime}$ are no longer small, and so strongly couple the two gap equations. The insensitivity of the superfluid gaps to $n_{0}^{+}$is a consequence of the large number of carriers in the conduction band excited from the valence band for all $n_{0}^{+}$. This means that the total number of carriers in the conduction band remains large for all $n_{0}^{+}$. Thus, the conduction band condensate remains trapped in the crossover regime and is unable to reach the BEC regime even when $n_{0}^{+}$becomes very small.

Thus, since $F_{\mathbf{k} \mathbf{k}^{\prime}}^{\gamma \gamma^{\prime}}$ controls the coupling of the two gap equations, the dependence of $F_{\mathbf{k k}^{\prime}}^{\gamma \gamma^{\prime}}$ on $E_{g}$ for $\gamma \neq \gamma^{\prime}$ means that by tuning $E_{g}$ we are able, for the first time, to tune the magnitude of the Josephson-like pair transfer.

Our neglect of cross-pairing is justified both for large and small gaps $E_{g}$. For large $E_{g}$ it is clear because of the large energy differences in the corresponding denominators. For small $E_{g}$, the large number of carriers in the conduction band excited from the valence band means a large effective Fermi energy, so the cross-pairing terms again contain large-energy-difference denominators, reflecting the large energy separation of the carriers in the valence band from the effective Fermi energy. In addition, the matrix elements for the cross-pairing terms are expected to be small (see Ref. [27]), and this would further reduce the cross-pairing contribution.

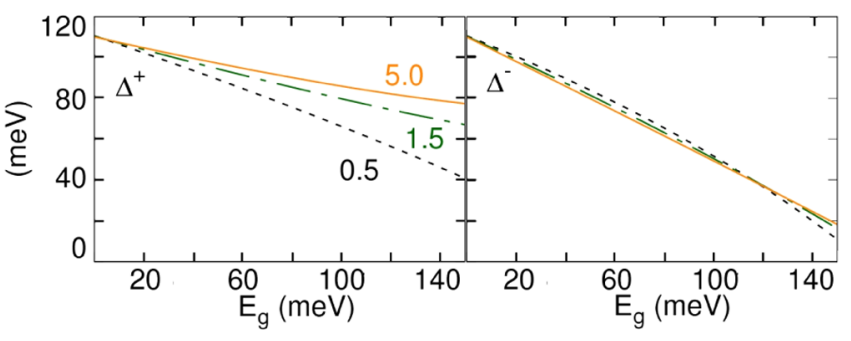

FIG. 4. Maximum superfluid gap energy $\Delta^{ \pm}$in the conduction and valence bands as functions of the energy band gap $E_{g}$. Dotted lines, $n_{0}^{+}=0.5 \times 10^{11} \mathrm{~cm}^{-2}$; dashed lines, $n_{0}^{+}=1.5 \times 10^{11} \mathrm{~cm}^{-2}$; solid lines, $n_{0}^{+}=5 \times 10^{11} \mathrm{~cm}^{-2}$.

Figure 4 further characterizes the multicomponent nature of the superfluidity. As expected, for zero band gap the maximum superfluid gap energy for the conduction band $\Delta^{+}$is equal to $\Delta^{-}$, the maximum superfluid gap for the valence band. We see in the figure that for smaller band gaps, $E_{g} \lesssim 80-120 \mathrm{meV}$, there is still a significant boost of both $\Delta^{+}$and $\Delta^{-}$, thanks to the multicomponent property that the contributions from the condensates are additive. For too large a band gap, the superfluidity will not be able to take advantage of a proximate valence band, and for $E_{g} \gtrsim 150 \mathrm{meV}$ the valence band condensate is essentially completely decoupled from the conduction band condensate. This results in $\Delta^{+} \gg \Delta^{-}$, so there is then only one significant superfluid gap and one significant condensate. Thus, continuously tuning $E_{g}$ up to higher values will induce, in the same system, a switching over of the number of superfluid components from 2 to 1 . However, for optimal conditions for superfluidity, the band gap $E_{g}$ must also not be too small; otherwise, excitations from the valence band will maintain too high a density of carriers in the conduction band. We recall, as we have discussed, that a high density of carriers inhibits superfluidity.

Thus, we conclude that a compromise is necessary between selecting too large an $E_{g}$, which tends to weaken the superfluidity since it excludes the additive contributions from the valence band, and too small an $E_{g}$, which tends to keep the conduction band in the high-density regime that is not favorable for superfluidity. An optimal choice would be in the range $E_{g} \sim 80-120 \mathrm{meV}$.

By using the tunable band gap $E_{g}$, we can move the boundaries of the BCS-BEC crossover while keeping the density fixed. When $\sum_{k}\left(v_{k}^{+}\right)^{2} \sim \sum_{k}\left(u_{k}^{-}\right)^{2}$ the multicomponent character is evident. There are two distinct regions: (i) For $E_{g} \ll E_{F}^{*}$, the small $n_{0}^{+}$region remains in the crossover regime even when $n_{0}^{+}$is very small. The conduction band condensate cannot enter the BEC regime because excitations from the valence band, equal to $g_{s} g_{v} \sum_{k}\left(u_{k}^{-}\right)^{2}$, maintain a large number of carriers in the conduction band. (ii) When $E_{g} \gtrsim E_{F}^{*}$, the conduction band condensate can enter the BEC regime for small $n_{0}^{+}$because a large $E_{g}$ suppresses excitations from the valence band. 
These multicomponent properties are reflected in the asymptotic behavior of the chemical potential in the small- $n_{0}^{+}$limit. Thus, for large $E_{g} \geq \varepsilon_{B}$, the limiting behavior of $\mu$ is the familiar BEC limit $\mu \rightarrow-\varepsilon_{B} / 2$, as for a single-component system, while for $E_{g}<\varepsilon_{B}$ the superfluid is blocked from entering the BEC regime even in the low-density limit; it is interesting that the limiting behavior of $\mu$ switches smoothly over to the midpoint of the band gap, $\mu \rightarrow-E_{g} / 2$.

We thank Mohammad Zarenia for useful discussions. Part of this work was supported by FWO-VI (Flemish Science Foundation) and the Methusalem program. *andrea.perali@unicam.it

[1] A. K. Geim and I. V. Grigorieva, Nature (London) 499, 419 (2013).

[2] K. Lee, J. Xue, D. C. Dillen, K. Watanabe, T. Taniguchi, and E. Tutuc, Phys. Rev. Lett. 117, 046803 (2016).

[3] J. I. A. Li, T. Taniguchi, K. Watanabe, J. Hone, A. Levchenko, and C.R. Dean, Phys. Rev. Lett. 117, 046802 (2016).

[4] X. Liu, K. Watanabe, T. Taniguchi, B. I. Halperin, and P. Kim, Nat. Phys. 13, 746 (2017).

[5] A. Perali, D. Neilson, and A. R. Hamilton, Phys. Rev. Lett. 110, 146803 (2013).

[6] Y. E. Lozovik and V. Yudson, Zh. Eksp. Teor. Fiz. 71, 738 (1976) [Sov. Phys. JETP 44, 389 (1976)].

[7] S. Shevchenko, Fiz. Nizk. Temp. 2, 505 (1976) [Sov. J. Low Temp. Phys. 2, 251 (1976)].

[8] K. Lee, B. Fallahazad, J. Xue, D. C. Dillen, K. Kim, T. Taniguchi, K. Watanabe, and E. Tutuc, Science 345, 58 (2014).

[9] Y. Zhang, T. T. Tang, C. Girit, Z. Hao, M. C. Martin, A. Zettl, M. F. Crommie, Y. R. Shen, and F. Wang, Nature (London) 459, 820 (2009).

[10] A. Bianconi, Nat. Phys. 9, 536 (2013).
[11] S. Rinott, K. Chashka, A. Ribak, E. D. Rienks, A. Taleb-Ibrahimi, P. Le Fevre, F. Bertran, M. Randeria, and A. Kanigel, Sci. Adv. 3, e1602372 (2017).

[12] See Supplemental Material at http://link.aps.org/ supplemental/10.1103/PhysRevLett.119.257002 for the model Hamiltonian, which includes Refs. [13,14].

[13] Y.E. Lozovik and A. Sokolik, Phys. Lett. A 374, 326 (2009).

[14] K. Zou, X. Hong, and J. Zhu, Phys. Rev. B 84, 085408 (2011).

[15] X. F. Wang and T. Chakraborty, Phys. Rev. B 81, 081402 (2010).

[16] Y. Barlas, T. Pereg-Barnea, M. Polini, R. Asgari, and A. H. MacDonald, Phys. Rev. Lett. 98, 236601 (2007).

[17] E. H. Hwang, R. Sensarma, and S. Das Sarma, Phys. Rev. B 84, 245441 (2011).

[18] L. Britnell, R. V. Gorbachev, R. Jalil, B. D. Belle, F. Schedin, M. I. Katsnelson, L. Eaves, S. V. Morozov, A. S. Mayorov, N. M. R. Peres et al., Nano Lett. 12, 1707 (2012); C. R. Dean, A. F. Young, I. Meric, C. Lee, L. Wang, S. Sorgenfrei, K. Watanabe, T. Taniguchi, P. Kim, K. Shepard et al., Nat. Nanotechnol. 5, 722 (2010); K. K. Kim, A. Hsu, X. Jia, S. M. Kim, Y. Shi, M. Dresselhaus, T. Palacios, and J. Kong, ACS Nano 6, 8583 (2012).

[19] P. Kumar, Y. S. Chauhan, A. Agarwal, and S. Bhowmick, J. Phys. Chem. C 120, 17620 (2016).

[20] R. Maezono, P. López Ríos, T. Ogawa, and R. J. Needs, Phys. Rev. Lett. 110, 216407 (2013).

[21] D. Neilson, A. Perali, and A. R. Hamilton, Phys. Rev. B 89, 060502 (2014).

[22] Y. E. Lozovik, S. L. Ogarkov, and A. A. Sokolik, Phys. Rev. B 86, 045429 (2012).

[23] X. Zhu, P. B. Littlewood, M. S. Hybertsen, and T. M. Rice, Phys. Rev. Lett. 74, 1633 (1995).

[24] P. Pieri, D. Neilson, and G. C. Strinati, Phys. Rev. B 75, 113301 (2007).

[25] L. Salasnich, N. Manini, and A. Parola, Phys. Rev. A 72, 023621 (2005); A. Guidini and A. Perali, Supercond. Sci. Technol. 27, 124002 (2014).

[26] G. Strinati, Phys. Essays 13, 427 (2000).

[27] L. Brey and H. A. Fertig, Phys. Rev. B 75, 125434 (2007). 